etiology of a variety of diseases, including DM and RA [1]. Additionally, cardiovascular and musculoskeletal complications from DM may influence the outcomes of RA patients.

Objectives: To investigate the impact of DM on outcomes of RA patients.

Methods: This is a cross-sectional study including 583 RA patients with 5 years' history after diagnosis in Tan Tock Seng Hospital RA registry, Singapore from 2001 to 2013. Information related to demographics, serologies, clinical features, comorbidities, and outcomes was collected. Independent t-test or Mann-Whitney $U$ test was used to compare continuous quantitative data, while Pearson Chi-square or Fisher Exact test for categorical data. With adjustment for age, gender, ethnicity, smoking and comorbidities, multivariate regressions were performed to analyze the impact of DM on outcomes of RA patients.

Results: DM is more prevalent in Malay and Indian patients than Chinese patients with RA $(26 \%, 24 \%$ and $11 \%$ respectively, $p=0.005)$. There is no difference of disease activity between DM and non DM patients. There is a tendency that non diabetic RA patients use more methotrexate $(p=0.052)$ and leflunomide $(p=0.058)$. Diabetic RA patients are in higher risk of poor American College of Rheumatology (ACR) functional status $(p=0.009)$, knee arthroplasty $(p<0.001)$ and admissions $(p=0.006)$. Adjusted for age, gender, ethnicity, smoking and comorbidities, multivariate regression analyses showed a trend of poor function status for diabetic RA patients, i.e. ACR functional status (adjusted odds ratio [aOR]: 1.802, 95\% confidence interval [Cl]: 0.968 - 3.353, $p=0.063$ ) and median Health Assessment Questionnaire (HAQ) ( $\beta$ coefficient value: $0.129,95 \% \mathrm{Cl}:-0.010-0.267, p=0.068)$, and higher risk for knee arthroplasty for diabetic RA patients (aOR: $3.480,95 \% \mathrm{Cl}: 1.016-11.920$, $p=0.047$ ).

Conclusion: This is the first report on the impact of DM on RA outcomes in a long term follow-up RA registry in a multiethnic Asian society.

References:

[1] Furman, D., Campisi, J., Verdin, E. et al. Chronic inflammation in the etiology of disease across the life span. Nat Med 25, 1822-1832 (2019).

Acknowledgments: TTSH Rheumatoid Arthritis Study Group

Disclosure of Interests: None declared

DOI: 10.1136/annrheumdis-2020-eular.5203

\section{AB0271 EFFECTIVENESS OF DULOXETINE FOR RELIEF OF THE REMNANT PAIN OF RHEUMATOID ARTHRITIS PATIENT WHOSE DISEASE ACTIVITY IS REMISSION}

I. Yoshii . ${ }^{1}$ Yoshii Hospital, Rheumatology and Musculoskeletal Medicine, Shimanto City, Japan

Background: Pain control in rheumatoid arthritis (RA) patient is an important matter. When pain remains even disease activity is remission, it causes deterioration of activity in daily living (ADL) in past research. In other words, pain affects ADL independently from disease activity, namely the Health Assessment Questionnaire (HAQ) score, a most popular index of ADL for patient with RA[1]. Thus, burden of remnant pain despite clinical remission in RA is serious and pending subject.

Duloxetine, a potent reuptake inhibitor of serotonin and norepinephrine, is developed for the treatment of major depressive disorder [2]. It's effectiveness for pain relief with osteoarthritis is also widely accepted. This drug should be effective not only for chronic pain due to osteoarthritis, but also due to RA. However, effectiveness of duloxetine for remnant pain relief in patient with RA in clinical remission is still unclear.

Objectives: In this study, effectiveness of duloxetine for the remnant pain despite clinical remission in patient with RA was statistically evaluated.

Methods: RA patients whose pain score with visual analog scale (PS-VAS) $>30 \mathrm{~mm}$ despite Clinical Disease Activity Score (CDAI) is $<2.8$, were picked up for the study. These patients were divided into groups whether duloxetine was administrated (a group without duloxetine: G-C; a group with duloxetine: G-D).

PS-VAS, C-reactive protein, CDAI and simplified disease activity index (SDAI), modified Health Assessment Questionnaire (mHAQ), and QOL value which is calculated from Euro-QOL 5-Dimensions (EQ-5D) were measured at the initiation of duloxetine in the G-D and at the first CDAI remission attained in the G-C, and at week 12 thereafter. Change of these indices were compared with One sample T-test for each group. Patient's global assessment (PGA) at baseline compared to the other components of CDAI was evaluated for each group statistically with One-tailed T-test.
Differences between the two groups at each moment were statistically evaluated with Mann-Whitney U-test. Statistical significance was set less than $1 \%$. All statistical analyses were performed using StatPlus:mac ${ }^{\circledR}$ (AnalystSoft Inc., Walnut, CA, USA).

Results: A total of three hundred and six patients were recruited. G-D counted sixty-eight with 18 males and 50 females, while G-C counted 238 with 57 males and 181 females. Average age were 71.3 and 71.5 for G-D and G-C respectively, with 53.6 months for time span from baseline to initiation in the G-D. $80.8 \%$ of the patients in G-D sustained to administrate duloxetine. PGA was 0.6 and 0.5 for $\mathrm{G}-\mathrm{D}$ and $\mathrm{G}-\mathrm{C}$ respectively, while the other component of CDAl were below 0.3 in average for both groups and these values were significantly lower than the PGA score in both groups. PS-VAS was 46.4 and 44.0, and significantly decreased to 26.1 and 36.0 in average for $G-D$ and G-C respectively at week 12 when compared to baseline. Reversely, the CDAI score was significantly elevated significantly from 1.16 and 1.19 to 3.25 and 4.34 for G-D and G-C respectively. PGA also significantly increased to 1.5 and 2.4 for G-D and G-C respectively. CRP and the SDAI score also demonstrated same trend significantly as the CDAI score for both groups. $\mathrm{mHAQ}$ decreased significantly from 0.430 and 0.495 to 0.393 and 0.487 for $G-D$ and $G-C$ respectively. QOL value increased from 0.800 and 0.817 to 0.811 and 0.840 for G-D and $\mathrm{G}-\mathrm{C}$ respectively, however no statistical significance demonstrated in both groups.

Conclusion: Duloxetine has been suggested to have effectiveness for the pain relief, for improvement of $A D L$, and for the contribution to QOL maintenance, however, no effect of disease activity control is expected.

\section{References:}

[1] Yoshii I, Chijiwa T, Sawada N. Influence of pain score measured by a visual analog scale (PS-VAS) on the Health Assessment Questionnaire Disability Index and 28-joint Disease Activity Index with C-reactive protein in rheumatoid arthritis patients. Int J Rheum Dis 2018;21:1955-61.

[2] Knadler MP, Lobo E, Chappell J, Bergstrom R. Duloxetine. Clin Pharmacokinet 2011;50:281-94.

Disclosure of Interests: None declared

DOI: 10.1136/annrheumdis-2020-eular.1370

\section{Rheumatoid arthritis - biological DMARDs}

\section{AB0272 ETANERCEPT BIOSIMILAR. EXPERIENCE IN A TERTIARY HOSPITAL.}

V. Aldasoro $^{1}$, J. Mendizabal ${ }^{1}$, S. Perez Garcia ${ }^{1}$, G. Sada Urmeneta $^{1}$

J. Restrepo Vélez ${ }^{1}$, N. Del Val del Amo ${ }^{1}$, I. Paniagua Zudaire ${ }^{1}$, R. Gutiérrez Polo ${ }^{1}$, L. Horcada ${ }^{1}$, L. Garrido Courel ${ }^{1}$, C. Fito-Manteca ${ }^{1} .{ }^{1}$ Navarra University Complex, Rheumatology, Pamplona, Spain

Background: With the arrival of biosimilar drugs and savings policies to make the health system sustainable, hospital managers have chosen to make changes from original molecules to biosimilar drugs.

Objectives: This work aims to reflect what happens when making these switchings.

Methods: We reviewed 235 patients who started Etanercept original in Rheumatology at Navarra Hospital Complex and Henares University Hospital and their switch to Etanercept biosimilar with a follow-up of 6 months

Results: The switch was performed in 174 patients with psoriatic arthritis (PsA) rheumatoid arthritis (RA), ankylosing spondylitis (AS), juvenile idiopathic arthritis, SAPHO and spondyloarthritis.

9.8\% discontinued treatment: 6 RA (8.1\%), 5 PsA (9.8\%) and 6 AS (20.7\%) all of them in the injection presentation. 12 patient stopped treatment due to ineffecacy, 2 due to reaction at the injection site, 2 due to diarrhea and 1 due to headache. Among $88.2 \%$ of patients who returned to Etanercept original, $28.6 \%$ did not achieve good response and had to change of treatment. The median time from Etanercept original beginning until the moment of switching was 54 (40-87) months.

Conclusion: In our series, approximately $10 \%$ of switching patients failed after a 6-month of follow-up; when trying to return to Etanercept original $28.6 \%$ did not achieve response.

The median persistence time in the original molecule and the percentage of failures observed in AS could be two conditions to consider before switching. A longer-term follow-up and a greater number of patients are necessary to ratify these data. 